$\mathrm{IC} / 93 / 33$

hep-th/9302100

\title{
STATIC QUARK POTENTIAL FROM THE POLYAKOV SUM OVER SURFACES
}

\author{
Zbigniew Jaskólski* \\ Krzysztof A. Meissner ${ }^{\dagger}$ \\ International Centre for Theoretical Physics, Trieste, Italy \\ February 17, 1993
}

\begin{abstract}
Using the Polyakov string ansatz for the rectangular Wilson loop we calculate the static potential in the semiclassical approximation. Our results lead to a well defined sum over surfaces in the range $1<d<25$.
\end{abstract}

*E-mail: jaskolsk@ictp.trieste.it. Permanent address: Institute of Theoretical Physics, Wrocław University, pl. Maxa Borna 9, 50-204 Wrocław, Poland

${ }^{\dagger}$ E-mail: meissner@ictp.trieste.it. Permanent address: Institute of Theoretical Physics, Warsaw University, Hoża 69, 00-681 Warszawa, Poland 


\section{Introduction}

In the present paper we address the question of the static $q \bar{q}$ - potential in the noncritical Polyakov string model [1] for the Wilson loop. The string calculation of this object stems from the old idea [2, 3] according to which the sum over world-sheets with fixed boundary provides a reasonable low energy approximation for the loop expectation value in QCD. The problem of the static potential in the Schild-Eguchi [4] and in the Nambu-Goto string models was solved several years ago [5, 6, 7, 8, 9]. Actually it is one of few string model predictions which can be compared with the Monte Carlo 10, 11] and the experimental [13 QCD data. Since the noncritical Polyakov string does not fall into the universality class [6] of models mentioned above it is desirable to have corresponding results also in this case. There is however a more general theoretical context which is in fact our main motivation. The long rectangular Wilson loop - an object with clear physical and geometrical interpretation, yet simple enough to make explicit calculations possible - is an ideal theoretical laboratory to investigate the Dirichlet type boundary conditions for the Polyakov sum over surface in noncritical dimensions. It seems that a better understanding of this problem can shed new light both on the Polyakov string ansatz for the loop equations and for the quantum physics of the conformal factor itself .

The first seminal calculations of the leading (1-loop) correction to the loop expectation value in the Schild-Eguchi model was done by Lüsher, Symanzik and Weisz [5]. In the particular case of long rectangular loop they discovered the Coulomblike correction to the linear static potential at large distances. It was subsequently observed by Lüscher [6] that this term is universal for all string models for which the transversal "embedding" variables are the only relevant degrees of freedom in the low energy limit. This is in contrast with the higher order corrections to the effective "transverse" action which are model dependent [7]. An attempt to probe small distances was done in [8], where the leading term in the $1 / d$ expansion was calculated in the Eguchi and in the Nambu-Goto models. This result exhibited the existence of a critical distance and was further confirmed by exact calculations in the light-cone operator quantization of the critical Nambu-Goto string [9]. These string model predictions were confronted with Monte Carlo data in a number of papers [10]. The general outcome of these analyses is a confirmation of the effective string picture at large distances and in particular the presence of the roughening phenomenon. The results concerning the numerical value of the coefficient of the Coulomb-like term depend on the methods used to disentangle finite size effects and seem to be insufficient to select a proper string model [11, 12].

The covariant functional techniques employed in the present paper are based on the discussion of the Polyakov string model for the Wilson loop due to O.Alvarez. In his pioneering paper [14] the boundary contributions to the conformal anomaly along with the explicit forms of integration measures for Teichmüller parameters and the conformal factor were calculated for bordered surfaces of arbitrary topological type. At that time however, the only available technique to deal with the Liouville sector was the saddle point approximation [15, 16]. A few years ago the main problem in the quantization of the Liouville theory - the field dependent functional measure - was successfully solved by David [17] and Distler and Kawai [18 within the CFT approach. The DDK method was soon rederived by direct calculations of the functional Jacobian [19, 20]. Since the approach of [19, 20] does not appeal to the conformal invariance of the combined ghost - Liouville - matter system it can be firmly used in the range $1<d<25$, where the CFT methods break down. This 
is one of important points in our presentation.

The second crucial issue is the choice of boundary conditions. For previous discussions of this point we refer to [14, 15, 16]. In the present paper we use the boundary conditions uniquely determined by the consistency conditions of the Faddeev-Popov procedure. They were first introduced in the context of off-shell critical string amplitudes [22] and recently analysed in the Liouville gravity coupled to the conformal matter in [23]. This choice is motivated by the interpretation of the Polyakov path integral as a sum over surfaces, which in the continuum formulation is based on the geometrical content of the Faddeev-Popov method.

The organization of the paper is as follows. In Section 2 we discussed the Polyakov sum over surfaces with particular attention paid to the proper treatment of the boundary conditions. In the case relevant for calculations of the static potential the formula for the Wilson loop is derived. As a result of our choice of boundary conditions one gets a novel approach to the Liouville theory and its interaction with the "matter" sector. In particular our formula for the Wilson loop does not contain the averaging over boundary reparametrisations [21] nor the integration over boundary values of conformal factor [16] although the second method may be equivalent to the one presented in this paper. The considerations of this section are general and can be applied to arbitrary contours and model manifolds. In Sect.3 we present all calculations which can be done exactly in the case of rectangular loop. For the vanishing and for the strictly positive value of the bulk cosmological constant $\mu$ one gets two different expressions containing path integrals over the nonconstant modes of conformal factor. Being unable to perform these integrals exactly we introduce an approximation scheme with the expansion parameter $\epsilon=\frac{\sqrt{2 \pi \alpha^{\prime}}}{R}$. The general features of this scheme are discussed in Sect. 4 in the case $\mu=0$. Since $\epsilon$ enters the effective action for $\phi$ only via the interaction term the coefficients in the corresponding power expansion get additional $\epsilon$-dependence. This also leads to a nonstandard relation with the loop expansion. In order to recover the static potential in the semiclassical approximation we analyse the limit of long rectangular loop. In this limit all functional integrals can be performed. Using the saddle point approximation of the resulting integral over the Teichmüller parameter we derive the explicit large $T$ asymptotic from which the static potential can be obtained. The corresponding calculations for $\mu>0$ are presented in Sect.5. On the semiclassical level the static potential is the same in both cases. The comparison with the predictions of string models from Lüscher's universality class suggests a simple (free) dynamics of the longitudinal mode. Some technical points of our derivation are explained in the appendix. Finally Sect.6 contains conclusions and some suggestions for future work.

\section{Boundary conditions}

We start with a brief description of the approach of [22] in the context of noncritical string. For simplicity we consider a loop $c \in R^{d}$ with four corners and the Polyakov path integral

$$
W[c]=\int_{\mathcal{F}[c]} \mathcal{D} g \mathcal{D} x(\text { VolDiff })^{-1} \exp -\frac{1}{4 \pi \alpha^{\prime}} S[g, x]
$$

over all surfaces of the topology of rectangle bounded by the loop $c$. The choice of loop with corners is slightly artificial from the point of view of the Wilson loop. We have chosen it to illustrate the general structure of the boundary conditions, 
first because it is directly related to the example we will calculate in the following; secondly it allows to avoid the technicalities related to the $S^{1}$ action present in the case of boundary components diffeomorphic to $S^{1}$ [24].

Let $M$ be a model rectangle with boundary components $\partial M_{i} ; i=1, \ldots, 4 ; \mathcal{M}_{M-}^{-}$ the space of all metrics on $M$, and $\mathcal{E}_{M}^{d}$ - the space of all maps $x: M \rightarrow R^{d}$. Our aim is to determine the subspace $\mathcal{F}[c] \subset \mathcal{M}_{M} \times \mathcal{E}_{M}^{d}$ of "string trajectories" bounded by the loop $c$ and satisfying the consistency conditions mentioned in the introduction. Clearly for any $(g, x) \in \mathcal{F}[c], x_{\mid \partial M}: \partial M \rightarrow R^{d}$ must be a parametrization of $c$. With our ability to perform only gaussian integrals we need the Dirichlet boundary conditions for $x$ with some choice of this parametrization. On the other hand in the next step we would like to use the F-P procedure with respect to the group Diff ${ }_{M}$ which means that the result of $x$-integration must be a $D i f f_{M}$-invariant functional of $g$. These two requirements are in apparent contradiction. Fortunately there is a simple solution for this puzzle. At the beginning we have tacitly assumed that each smooth component $c_{i}, i=1, \ldots, 4$ of $c$ is a point in the space

$$
c_{i} \in \frac{\mathcal{E}_{I_{i}}^{d}}{\operatorname{Diff}_{I_{i}}}
$$

where $\mathcal{E}_{I_{i}}^{d}$ is the space of maps $\widetilde{x}: I_{i} \rightarrow R^{d}$ and $I_{i}, i=1, \ldots, 4$ are model intervals. Suppose $c$ has an additional internal structure, namely

$$
c_{i} \in \overline{\mathcal{C}}_{i} \equiv \frac{\mathcal{M}_{I_{i}} \times \mathcal{E}_{I_{i}}^{d}}{\operatorname{Diff}_{I_{i}} \times R_{+}} \approx \mathcal{E}_{I_{i}}^{d},
$$

where $\mathcal{M}_{I_{i}}$ is the space of all einbeins on $I_{i}$ and $R_{+}$stands for the group of constant rescalings of einbeins. (If $c_{i}=\left[\left(\widetilde{e}_{i}, \widetilde{x}_{i}\right)\right]$ and $\widetilde{x} \in \mathcal{E}_{I_{i}}^{d}$ is regular enough one can regard $c_{i}$ as a 1-dim submanifold of $R^{d}$ endowed with intrinsic metric determined up to a constant multiplicative factor.) Now let us observe that each "string trajectory" $(g, x) \in \mathcal{F}[c]$ uniquely determines a point

$$
\left[\left(e_{i}, x_{i}\right)\right] \in \frac{\mathcal{M}_{\partial M_{i}} \times \mathcal{E}_{\partial M_{i}}^{d}}{\operatorname{Diff}_{\partial M_{i}} \times R_{+}},
$$

where $e_{i}$ denotes the einbein induced by $g$ on $\partial M$ and $x_{i}=x_{\mid \partial M_{i}}$. Since the spaces (3),(迹) are canonically isomorphic, for any system of $c_{i} \in \mathcal{C}_{i}$ (satisfying appropriate compatibility conditions at the ends of $I_{i}$ ) the boundary conditions

$$
\left[\left(e_{i}, x_{i}\right)\right]=c_{i} \quad, \quad i=1, \ldots, 4 .
$$

are perfectly well defined and $D i f f_{M}$-invariant. For each metric $g$ these conditions imply a $g$-dependent Dirichlet boundary condition for $x$ [22]. Hence the $x$ integration is gaussian and yields Diff $_{M^{-}}$-invariant functional $Z[c, g]$ on $\mathcal{M}_{M}$

$$
Z[c, g]=\left(\operatorname{det} \mathcal{L}_{g}\right)^{-\frac{d}{2}} \exp -S_{\text {eff }}[c, g],
$$

where the determinant for the scalar Laplace-Beltrami operator is calculated for the homogeneous Dirichlet boundary condition.

Two remarks concerning the boundary conditions (5) are in order. First of all they are not invariant with respect to general Weyl transformations. This fact leads in the critical string theory to the gauge dependence of the off-shell amplitudes. In the noncritical theory it yields nontrivial coupling of the conformal factor to the 
matter fields via boundary conditions. This was first pointed out in [16] where a slightly stronger version of the boundary condition (5) was proposed. Secondly the Wilson loop $c$ in QCD is supposed to be a "nonparametrized contour" i.e. a point in the space (2). Switching over from (2) to (3) means introducing some preferred parametrization of $c$. When the loop $c$ is regular enough (locally embedded in $R^{d}$ ) there exists a natural choice - the parametrization in which the metric induced by the target space metric is constant. We will use this parametrization for the rectangular loop appearing in calculations of the static potential. Note that for less regular loops this simple prescription breaks down.

The next step in the calculation of (11) is to apply the F-P method to the path integral

$$
W[c]=\int_{\mathcal{M}_{M}^{*}} \mathcal{D} g(\text { VolDiff })^{-1} Z[c, g],
$$

where the integration domain $\mathcal{M}_{M}^{*} \subset \mathcal{M}_{M}$ is not yet specified. The problem of functional integration over the space of metrics on a bordered surface with Diff invariant integrand has been recently analysed in [23]. It was shown that the consistency of the F-P procedure uniquely determines a family of admissible integration domains $\mathcal{M}_{M}^{n *} \subset \mathcal{M}_{M}$ parametrized by the space of all normal directions $n$ on the boundary. Moreover the resulting path integral is independent on $n$ which means that $\mathcal{M}_{M}^{*}$ in the formula (6) is in fact uniquely determined.

In the case under consideration, for a fixed normal direction $n$ on $\partial M$ the space $\mathcal{M}_{M}^{n *}$ can be described as follows. Let $M^{D D}$ be the double double of $M$ (i.e. the result of subsequent doubling with respect to two pairs of disjoint boundary components of the rectangle $M$ ) and $i, j$ - corresponding involutions of $M^{D D}$ with the invariant direction $n$ on $\partial M$. Then the space $\mathcal{M}_{M}^{n *}$ consists of all metrics on $M$ admitting a $C^{1}$-extension to an $i, j$-symmetric metric on $M^{D D}$. Another description of $\mathcal{M}_{M}^{n *}$ can be given in terms of the space $\mathcal{M}_{M}^{n o}$ of all metrics on $M$ with the normal direction $n_{g}=n$ and the scalar curvature $R_{g}=0$ such that all boundary components are geodesic and meet orthogonally. One can show that $\mathcal{M}_{M}^{n *}$ consists of all metrics of the form $e^{\phi} g_{o}$ where $g_{o} \in \mathcal{M}_{M}^{n o}$ and $\phi$ satisfies the Neumann boundary condition $n^{a} \partial_{a} \phi=0$ on $\partial M$. The most important consequence of this result is that in every conformal gauge $R_{+} \ni t \rightarrow \widehat{g}_{t} \in \mathcal{M}_{M}^{n *}, g=e^{\phi} \widehat{g}_{t}$ the conformal factor satisfies the Neumann boundary condition

$$
n^{a} \partial_{a} \phi=0
$$

on each boundary component.

With the choice of $\mathcal{M}_{M}^{n *}$ as an integration domain in (6) the F-P procedure in a conformal gauge yields

$$
\begin{aligned}
W[c]=\int_{0}^{\infty}[d t] \int_{\mathcal{W}^{n}} \mathcal{D}^{\widehat{g}_{t}} \phi & \times \exp \left(-\frac{25-d}{48 \pi} S_{L}\left[\widehat{g}_{t}, \phi\right]\right) \\
& \times \exp \left(\frac{7+d}{32} \sum_{\text {corners }} \phi\left(z_{i}\right)\right) \\
& \times \exp \left(-\frac{1}{4 \pi \alpha^{\prime}} S_{\text {eff }}\left[c, e^{\phi} \widehat{g}_{t}\right]\right)
\end{aligned}
$$

where $[d t]$ stands for the $\phi$-independent part of the integrand and $\mathcal{W}^{n}$ is the space of all realvalued functions on $M$ satisfying the boundary condition (7). For the calculation of the corner contribution to the conformal anomaly we refer to the 
original paper 25] where this formula was first derived. As a consequence of our choice of integration domain in (6) the Liouville action in (8)

$$
S_{L}[g, \varphi]=\int_{M_{h, b}} d^{2} z \sqrt{g}\left(\frac{1}{2} g^{a b} \partial_{a} \varphi \partial_{b} \varphi+R_{g} \varphi+\mu e^{\varphi}\right)
$$

does not contain boundary terms. In particular the boundary conditions (]) imply the vanishing boundary cosmological constant. The action is bounded from below for the bulk cosmological constant $\mu \geq 0$.

\section{$3 \quad$ Rectangular Wilson loop}

In order to make our abstract discussion more concrete let us introduce the 2dimensional

$$
\left(\widehat{M}_{t}, \widehat{g}_{t}\right)=\left([0, t] \times[0,1],\left(\begin{array}{ll}
1 & 0 \\
0 & 1
\end{array}\right)\right),
$$

and the 1-dimensional

$$
\begin{aligned}
& \left(\widehat{I}_{i}=\partial \widehat{M}_{t i}, \widehat{e}_{i}\right)=([0, t], 1) \quad i=1,3, \\
& \left(\widehat{I}_{i}=\partial \widehat{M}_{t i}, \widehat{e}_{i}\right)=([0,1], 1) \quad i=2,4 \quad
\end{aligned}
$$

conformal gauges. The measure $[d t]$ involving determinants of $P_{\hat{g}_{t}}^{+} P_{\hat{g}_{t}}$ and $\mathcal{L}_{\hat{g}_{t}}$ can be calculated by standard methods. In the conformal gauge (9) one gets [21, 25]

$$
[d t]=\eta(t)^{1-\frac{d}{2}} d t
$$

where

$$
\eta(t)=\mathrm{e}^{-\frac{\pi t}{12}} \prod_{n=1}^{\infty}\left(1-\mathrm{e}^{-2 \pi n t}\right) .
$$

Let $\left(\widetilde{x}_{i}, 1\right)$ be the representant of $c_{i}$ in the gauge $(10)$. According to 22

$$
S_{\mathrm{eff}}\left[c, e^{\phi} \widehat{g}_{t}\right]=S\left[\widehat{g}_{t}, \widetilde{x}_{i}[\phi]\right]=\int_{0}^{t} d z^{0} \int_{0}^{1} d z^{1}\left(\left(\partial_{0} x_{c l}\right)^{2}+\left(\partial_{1} x_{c l}\right)^{2}\right)
$$

where $x_{c l}: \widehat{M}_{t} \rightarrow R^{d}$ is the solution of the boundary value problem

$$
\begin{aligned}
& \left(\partial_{0}^{2}+\partial_{1}^{2}\right) x=O \\
& x_{\mid \partial M_{i}}=\widetilde{x}_{i}[\phi] \equiv \widetilde{x}_{i} \circ \gamma_{i}[\phi] \quad, i=1, \ldots, 4 .
\end{aligned}
$$

The diffeomorphisms $\gamma_{i}[\phi]:[0, t] \rightarrow[0, t], i=1,3$ and $\gamma_{i}[\phi]:[0,1] \rightarrow[0,1], i=2,4$ are uniquely determined by the equations

$$
\begin{array}{ll}
\frac{d}{d z^{0}} \gamma_{1}[\phi]\left(z^{0}\right) & \propto \exp \frac{1}{2} \phi\left(z^{0}, 0\right) \\
\frac{d}{d z^{1}} \gamma_{2}[\phi]\left(z^{1}\right) & \propto \exp \frac{1}{2} \phi\left(0, z^{1}\right), \\
\frac{d}{d z^{0}} \gamma_{3}[\phi]\left(z^{0}\right) & \propto \exp \frac{1}{2} \phi\left(z^{0}, 1\right), \\
\frac{d}{d z^{1}} \gamma_{4}[\phi]\left(z^{1}\right) & \propto \exp \frac{1}{2} \phi\left(t, z^{1}\right) .
\end{array}
$$

In general the prescription given above yields the functional $S\left[\widehat{g}_{t}, \widetilde{x}_{i}[\phi]\right]$ depending on the boundary value of $\phi$ in a very complicated way. The situation is more tractable 
for rectangular loops. Let $c_{R T}$ be a loop of width $R$ and length $T$ given in the 1-dim conformal gauge (10) by

$$
\begin{array}{rlrl}
\widetilde{x}_{1}^{0}\left(z^{0}\right) & =\frac{T}{t} z^{0}, & \widetilde{x}_{1}^{1}\left(z^{0}\right) & =0, \\
\widetilde{x}_{2}^{0}\left(z^{1}\right) & =0, & \widetilde{x}_{2}^{1}\left(z^{1}\right) & =R z^{1}, \\
\widetilde{x}_{3}^{0}\left(z^{0}\right) & =R, \\
\left.\widetilde{x}_{4}^{0}\right) & =\frac{T}{t} z^{0}, \\
\widetilde{x}_{4}^{0}\left(z^{1}\right) & =T, \\
\widetilde{x}_{i}^{k} \equiv 0 \quad \text { for } \quad i=1, \ldots, 4 ; k=2, \ldots, d-1 & =R z^{1},
\end{array}
$$

Solving the boundary value problem (12) and inserting solution into the Polyakov action one gets

$$
\begin{aligned}
S\left[\widehat{g}_{t}, \widetilde{x}_{i}[\phi]\right] & =\frac{T^{2}}{t}+2 T^{2} \sum_{n>0} \frac{1}{\pi n \sinh \frac{\pi n}{t}}\left[\left(f_{1 n}{ }^{2}+f_{3 n}{ }^{2}\right) \cosh \frac{\pi n}{t}-2 f_{1 n} f_{3 n}\right] \\
& +R^{2} t+2 R^{2} \sum_{m>0} \frac{1}{\pi m \sinh \pi m t}\left[\left(f_{2 m^{2}}{ }^{2}+f_{4 m}{ }^{2}\right) \cosh \pi m t-2 f_{2 m} f_{4 m}\right]
\end{aligned}
$$

where

$$
\begin{aligned}
f_{1 n} & =\frac{\pi n}{T t} \int_{0}^{t} d z^{0}\left(\frac{T}{t} \gamma_{1}[\phi]\left(z^{0}\right)-\frac{T}{t} z^{0}\right) \sin \frac{\pi n z^{0}}{t} \\
& =\left(\int_{0}^{t} d z^{0} \exp \frac{1}{2} \phi\left(z^{0}, 0\right)\right)^{-1} \int_{0}^{t} d z^{0} \exp \frac{1}{2} \phi\left(z^{0}, 0\right) \cos \frac{\pi n z^{0}}{t} \\
f_{2 m} & =\left(\int_{0}^{1} d z^{1} \exp \frac{1}{2} \phi\left(0, z^{1}\right)\right)^{-1} \int_{0}^{1} d z^{1} \exp \frac{1}{2} \phi\left(0, z^{1}\right) \cos \pi m z^{1} \\
f_{3 n} & =\left(\int_{0}^{t} d z^{0} \exp \frac{1}{2} \phi\left(z^{0}, 1\right)\right)^{-1} \int_{0}^{t} d z^{0} \exp \frac{1}{2} \phi\left(z^{0}, 1\right) \cos \frac{\pi n z^{0}}{t} \\
f_{4 m} & =\left(\int_{0}^{1} d z^{1} \exp \frac{1}{2} \phi\left(t, z^{1}\right)\right)_{0}^{1} d z^{1} \exp \frac{1}{2} \phi\left(t, z^{1}\right) \cos \pi m z^{1}
\end{aligned}
$$

Expanding the Liouville field $\phi$ in modes on $\widehat{M}_{t}$

$$
\phi=\phi_{0}+\frac{2}{\sqrt{t}} \sum_{m, n \geq 0}{ }^{\prime} \phi_{n m} \cos \frac{\pi n z^{0}}{t} \cos \pi m z^{1}
$$

one finally has

$$
\begin{aligned}
W\left[c_{R T}\right]= & \int_{0}^{\infty} d t \eta(t)^{1-\frac{d}{2}} \int_{-\infty}^{+\infty} \sqrt{t} d \phi_{0} \int \prod_{n, m \geq 0}^{\prime} d \phi_{n m} \\
& \times \exp \left(-\frac{25-d}{96 \pi}\left(2 \sum_{m \text { or } n=0}+\sum_{n, m \geq 1}\right)\left[\left(\frac{n^{2} \pi^{2}}{t^{2}}+m^{2} \pi^{2}\right) \phi_{n m}{ }^{2}\right]\right)
\end{aligned}
$$




$$
\begin{aligned}
& \times \exp \left(-\frac{1}{4 \pi \alpha^{\prime}} S\left[\widehat{g}_{t}, \widetilde{x}_{i}[\phi]\right]\right) \\
& \times \exp \left(\frac{7+d}{4 \sqrt{t}} \sum_{n, m \text { even }}{ }^{\prime} \phi_{n m}+\frac{7+d}{8} \phi_{0}\right) \\
& \times \exp \left(-\mu \mathrm{e}^{\phi_{0}} \int d^{2} z \exp \left(\frac{2}{\sqrt{t}} \sum_{m, n \geq 0}{ }^{\prime} \phi_{n m} \cos \frac{\pi n z^{0}}{t} \cos \pi m z^{1}\right)\right)
\end{aligned}
$$

where $S\left[\widehat{g}_{t}, \widetilde{x}_{i}[\phi]\right]$ is given by the equations (15), (16).

Since the boundary conditions (5) are invariant with respect to Weyl transformations with a conformal factor constant along boundary, the zero mode $\phi_{0}$ enters the effective action only through the corner anomaly and the Liouville interaction terms. In both cases $\mu=0, \mu>0$ the integration over $\phi_{0}$ can be performed exactly.

For $\mu=0, \phi_{0}$ couples only via the corner anomaly term which is independent of the Teichmüller parameter and of the parameters of $c_{R T}$. Hence the only contribution of $\phi_{0}$ is the term $\sqrt{t}$ coming from the $t$-dependence of the zero mode integration measure and some infinite constant which can be absorbed into an overall normalization factor. Up to this constant one has

$$
W\left[c_{R T}\right]=\int_{0}^{\infty} d t \eta(t)^{1-\frac{d}{2}} \sqrt{t} Z(R, T ; t)
$$

where

$$
Z(R, T ; t)=\int \mathcal{D}^{\prime} \phi \exp \left(-\frac{b}{2 \pi^{2}} \int d^{2} z(\nabla \phi)^{2}+\frac{c}{4} \sum_{\text {corners }} \phi\left(z_{i}\right)-\frac{1}{\epsilon^{2}} V[\widetilde{\phi}]\right),
$$

In the formula above, prime in the symbol of measure means that the integration is over all fields orthogonal to the zero mode subspace and

$$
\begin{aligned}
b & =\frac{(25-d) \pi}{48}, \\
c & =\frac{7+d}{8}, \\
V[\widetilde{\phi}] & =\frac{1}{2 R^{2}} S\left[\widehat{g}_{t}, \widetilde{x}_{i}[\phi]\right] \quad, \quad \widetilde{\phi}=\phi_{\mid \partial M} .
\end{aligned}
$$

In the presence of the exponential Liouville term in the action $(\mu>0)$, the integration over $\phi_{0}$ can be performed by means of the standard techniques [26, 27]. Using the formula

$$
\int_{-\infty}^{\infty} d \phi_{0} \exp \left(c \phi_{0}-\alpha \mathrm{e}^{\phi_{0}}\right)=\Gamma(c) \mathrm{e}^{-c \ln \alpha}
$$

one gets

$$
\begin{aligned}
W\left[c_{R T}\right] & =\mu^{-c} \Gamma(c) \int_{0}^{\infty} d t \eta(t)^{1-\frac{d}{2}} t^{-c} Z(R, T ; t) \\
Z(R, T ; t)=\int \mathcal{D}^{\prime} \phi & \exp \left(-\frac{b}{2 \pi^{2}} \int d^{2} z(\nabla \phi)^{2}+\frac{c}{4} \sum_{\text {corners }} \phi\left(z_{i}\right)-\frac{1}{\epsilon^{2}} V[\widetilde{\phi}]\right) \\
\times & \exp \left(-c \ln \left(\frac{1}{t} \int d^{2} z \exp (\phi)\right)\right) .
\end{aligned}
$$


Despite a considerable simplification in the $\phi$-dependence of $V[\widetilde{\phi}]$ due to the linear structure of boundary conditions (14), the "potential" part of the effective action is extremely complicated. Actually it is nonanalytical in terms of modes $\phi_{n m}$. However one can easily show by explicit calculations that the functional $V[\widetilde{\phi}]$ has a true minimum at $\phi_{n m} \equiv 0$ which makes semiclassical calculations possible.

\section{Semiclassical Approximation for $\mu=0$}

The special feature of the Polyakov model making the semiclassical calculations nontrivial is the universality of the coefficient in front of the Liouville action. Since it is a dimensionless constant uniquely determined by the number of target space dimensions, the only available "loop" expansion in this sector corresponds to the formal limit $d \rightarrow-\infty$ [16]. On the other hand the peculiar form of the boundary conditions (12),(13) and the decoupling of the zero mode from the $x$-sector lead to the conclusion that the conformal factor should be regarded as an infinite dimensional "Teichmüller parameter" rather than a physical degree of freedom. With this interpretation an appropriate expansion parameter for a physically meaningful semiclassical approximation is $\epsilon \equiv \frac{\sqrt{2 \pi \alpha^{\prime}}}{R}$.

In this section we analyse in more detail the semiclassical approximation in the case $\mu=0$. The corresponding consideration for the nonvanishing Liouville interaction are presented in Sect.5. In the case of rectangular loop the "potential"

term has an absolute minimum $\widetilde{\phi}_{c l} \equiv 0$ which coincides with the boundary value of the minimum of kinetic term. One can expect that in the limit $\epsilon \rightarrow 0$ the main contribution to the functional integral (19) comes from fluctuations around the classical configuration $\phi \equiv 0$. Inserting the formal expansion

$$
V[\widetilde{\phi}]=V\left[\widetilde{\phi}_{c l}\right]+\sum_{n \geq 2} \frac{1}{n !} V^{(n)}[\widetilde{\phi}]
$$

into (19) and changing variables $\phi \rightarrow \epsilon \phi$ one gets

$$
\begin{aligned}
Z(R, T ; t)= & \exp \left(-\frac{1}{\epsilon^{2}} V\left[\widetilde{\phi}_{c l}\right]\right) \\
\times & \int \mathcal{D}^{\prime} \phi \exp \left(-\frac{\epsilon^{2} b}{2 \pi^{2}} \int d^{2} z(\nabla \phi)^{2}+\frac{\epsilon c}{4} \sum_{\text {corners }} \phi\left(z_{i}\right)-\frac{1}{2} V^{(2)}[\widetilde{\phi}]\right) \\
& \times \exp \left(-\frac{\epsilon}{3 !} V^{(3)}[\widetilde{\phi}]-\frac{\epsilon^{2}}{4 !} V^{(4)}[\widetilde{\phi}]-\ldots\right) .
\end{aligned}
$$

The standard reasoning would lead to the conclusion that the 1-loop approximation is given by dropping all terms with positive powers of $\epsilon$. The resulting gaussian integral is, however, strongly divergent and requires some regularization procedure. Our method to deal with this problem consists in retaining at the 1-loop level all terms quadratic and linear in $\phi$. Thus, the 1-loop approximation is given by

$$
\begin{aligned}
Z_{1 \text { loop }}(R, T ; t) & =\exp \left(-\frac{1}{\epsilon^{2}} V\left[\widetilde{\phi}_{c l}\right]\right) \\
& \times \int \mathcal{D}^{\prime} \phi \exp \left(-\frac{\epsilon^{2} b}{2 \pi^{2}} \int d^{2} z(\nabla \phi)^{2}+\frac{\epsilon c}{4} \sum_{\text {corners }} \phi\left(z_{i}\right)-\frac{1}{2} V^{(2)}[\widetilde{\phi}]\right)
\end{aligned}
$$

The higher loop corrections are then defined by appropriate Wick contractions of the interaction terms in (23) calculated in the gaussian model (24). This prescription 
slightly differs from the usual loop expansion. In fact, due to the presence of higher order terms in (24) a fixed $n$-loop correction may contribute to several terms in the $\epsilon$-expansion. Moreover this additional $\epsilon$-dependence may destroy the power expansion we would like to develop. The basic consistency condition of the loop expansion given by (24) requires that contributions from higher corrections lead to a formal expansion of the following form:

$$
Z(R, T ; t)=\exp \left(-\frac{1}{\epsilon^{2}}\left[V\left[\widetilde{\phi_{c l}}\right]+\sum_{n \geq 1} \epsilon^{n} f_{n}(\epsilon)\right]\right),
$$

where $f_{n}(\epsilon)$ have at most logarithmic dependence on $\epsilon$ for all $n \geq 1$. When this condition is satisfied the semiclassical approximation is given by

$$
Z_{\text {s.c. }}(R, T ; t)=\exp \left[-\frac{1}{\epsilon^{2}}\left(V\left[\widetilde{\phi_{c l}}\right]+\epsilon f_{1}(\epsilon)\right)\right] .
$$

In the rest of this section we shall analyse the 1-loop correction in the limit of long rectangular loop. The interpretation of our result as a semiclassical approximation is based on the assumption that the condition (25) is satisfied and that the only contribution to the coefficient $f_{1}(\epsilon)$ comes from the 1-loop correction (24). The explicit calculations presented below suggest that in the limit $\frac{T}{R} \rightarrow \infty$ this assumption is well justified. An exact proof, however, requires a systematic analysis of higher order corrections and is beyond the scope of the present paper.

Expanding the functional (15) to the second power in the Liouville field around $\phi_{c l} \equiv 0$ and using explicit formula (17) one gets

$$
Z_{1 \text {-loop }}(R, T ; t)=e^{-\frac{1}{2 \epsilon^{2}}\left(\frac{T^{2}}{R^{2} t}+t\right)} \int \prod_{n, m \geq 0}^{\prime} d \phi_{n m} e^{-S_{1-\mathrm{loop}}[\phi]},
$$

where

$$
\begin{aligned}
S_{1 \text { loop }}[\phi] & =\frac{\epsilon^{2} b}{2}\left(2 \sum_{m \text { or } n=0}+\sum_{n, m \geq 1}\right)\left[\left(\frac{n^{2}}{t^{2}}+m^{2}\right) \phi_{n m}^{2}\right] \\
& +\frac{2 \epsilon c}{\sqrt{t}} \sum_{n, m \text { even }}{ }^{2} \phi_{n m} \\
& +\frac{T^{2}}{2 t R^{2}} \sum_{n=1}^{\infty}\left[\frac{\tanh \frac{\pi n}{2 t}}{\pi n}\left(\sum_{m \text { even }} \phi_{n m}\right)^{2}+\frac{\operatorname{coth} \frac{\pi n}{2 t}}{\pi n}\left(\sum_{m \text { odd }} \phi_{n m}\right)^{2}\right] \\
& +\frac{1}{2 t} \sum_{m=1}^{\infty}\left[\frac{\tanh \frac{\pi m t}{2}}{\pi m}\left(\sum_{n \text { even }} \phi_{n m}\right)^{2}+\frac{\operatorname{coth} \frac{\pi m t}{2}}{\pi m}\left(\sum_{n \text { odd }} \phi_{n m}\right)^{2}\right] .
\end{aligned}
$$

It will be shown in the Appendix A that the conformal anomaly (the second factor in equation (28) does not contribute to the result in the limit $\frac{T}{R} \rightarrow \infty$ and that the last sum in (28) can also be neglected. Hence, in the case of long rectangular loop the 1-loop effective action can be replaced in the formula (27) by

$$
\begin{aligned}
S_{1 \text {-loop }}^{\prime}[\phi] & =\frac{\epsilon^{2} b}{2}\left(2 \sum_{m \text { or } n=0}+\sum_{n, m \geq 1}\right)\left[\left(\frac{n^{2}}{t^{2}}+m^{2}\right) \phi_{n m}{ }^{2}\right] \\
& +\frac{T^{2}}{2 t R^{2}} \sum_{n=1}^{\infty}\left[\frac{\tanh \frac{\pi n}{2 t}}{\pi n}\left(\sum_{m \text { even }} \phi_{n m}\right)^{2}+\frac{\operatorname{coth} \frac{\pi n}{2 t}}{\pi n}\left(\sum_{m \text { odd }} \phi_{n m}\right)^{2}\right] .
\end{aligned}
$$


The resulting integral can be decomposed into an infinite product of gaussian integrals corresponding to each fixed value of the index $n$. The integration over the modes $\left\{\phi_{0, m}\right\}_{m \geq 1}$ yields just a constant factor. For every $n \geq 1$ the integration over $\left\{\phi_{n, m}\right\}_{m \geq 0}$ can be done by means of the formula

$$
\operatorname{det}\left(\begin{array}{cccc}
\alpha_{1}+\beta & \beta & \ldots & \beta \\
\beta & \alpha_{2}+\beta & \ldots & \beta \\
\ldots & \ldots & \ldots & \ldots \\
\beta & \beta & \ldots & \alpha_{n}+\beta
\end{array}\right)=\left(1+\sum_{j} \frac{\beta}{\alpha_{j}}\right) \prod_{i=1}^{n} \alpha_{i} .
$$

Performing all integrations one gets

$$
Z_{1 \text {-loop }}(R, T ; t) \approx e^{-\frac{1}{2 \epsilon^{2}}\left(\frac{T^{2}}{R^{2} t}+t\right)} \times I \times I_{o} \times I_{e},
$$

where the infinite products $I, I_{e}, I_{o}$ are given by

$$
\begin{aligned}
I & =\left[\prod_{n, m \geq 0} \frac{\epsilon^{2} b}{2}\left(\frac{n^{2}}{t^{2}}+m^{2}\right)\right]^{-\frac{1}{2}}=t^{-\frac{1}{2}} \eta(t)^{-\frac{1}{2}}, \\
I_{e} & =\prod_{n \geq 1}\left(1+\frac{T^{2} \tanh \frac{\pi n}{2 t}}{\epsilon^{2} b R^{2} \pi n t}\left(\frac{t^{2}}{2 n^{2}}+\sum_{m \text { even } \geq 2} \frac{1}{\frac{n^{2}}{t^{2}}+m^{2}}\right)\right)^{-\frac{1}{2}}, \\
I_{o} & =\prod_{n \geq 1}\left(1+\frac{T^{2} \operatorname{coth} \frac{\pi n}{2 t}}{\epsilon^{2} b R^{2} \pi n t} \sum_{m \text { odd }} \frac{1}{\frac{n^{2}}{t^{2}}+m^{2}}\right)^{-\frac{1}{2}} .
\end{aligned}
$$

Using

$$
\begin{gathered}
\frac{1}{2 a^{2}}+\sum_{k_{\text {even }} \geq 2} \frac{1}{k^{2}+a^{2}}=\frac{\pi}{4 a} \operatorname{coth}(\pi a / 2) \\
\sum_{k_{\text {odd }}>0} \frac{1}{k^{2}+a^{2}}=\frac{\pi}{4 a} \tanh (\pi a / 2)
\end{gathered}
$$

we find

$$
\begin{aligned}
& \ln I_{o}=-\frac{1}{2} \sum_{n} \ln \left(1+\frac{T^{2}}{4 \epsilon^{2} b R^{2} n^{2}}\right), \\
& \ln I_{e}=-\frac{1}{2} \sum_{n} \ln \left(1+\frac{T^{2}}{4 \epsilon^{2} b R^{2} n^{2}}\right) .
\end{aligned}
$$

Using the exact formula (which can be proven by differentiating the both sides over $\alpha)$

$$
\sum_{n \geq 1} \ln \left(1+\frac{\alpha^{2}}{n^{2}}\right)=\pi \alpha-\ln 2 \pi \alpha+\ln \left(1-e^{-2 \pi \alpha}\right)
$$

one gets the exact result for the series (33) and (34). Hence we can write

$$
\ln I_{o}=\ln I_{e}=-\frac{\pi T}{4 \sqrt{b} \epsilon R}+O\left(\ln \frac{\pi T}{\sqrt{b} \epsilon R}\right)
$$

Collecting all the results one gets in the limit $\frac{T}{R} \rightarrow \infty$

$$
Z_{1 \text {-loop }}(R, T ; t) \approx t^{-\frac{1}{2}} \eta(t)^{-\frac{1}{2}} \exp \left[-\frac{1}{\epsilon^{2}}\left(\frac{T^{2}}{2 R^{2} t}+\frac{t}{2}+\epsilon f_{1}^{1-\text { loop }}+\epsilon^{2} f_{2}^{1-\text { loop }}\right)\right],
$$


where

$$
\begin{aligned}
f_{1}^{1-\text { loop }}(\epsilon) & =\frac{2 \pi T}{4 \sqrt{b} R}, \\
f_{2}^{1-\text { loop }}(\epsilon) & =O\left(\ln \left(\frac{T}{\epsilon R}\right)\right) .
\end{aligned}
$$

The results above show that the consistency condition (25) is satisfied on the 1-loop level. Thus, under the assumption $f_{1}(\epsilon)=f_{1}^{1-l o o p}(\epsilon)$, the semiclassical approximation is given by dropping in the formula (37) all terms proportional to $\epsilon^{2}$. Note that these terms do not contribute to the static potential anyhow.

Inserting (37) into (18), changing variables $t \rightarrow \tau=\frac{t}{T}$ and neglecting all $O\left(\ln \left(\frac{T}{\sqrt{2 \pi \alpha^{\prime}}}\right)\right)$-terms in the exponent one gets

$$
W\left[c_{R T}\right] \approx \int_{0}^{\infty} d \tau e^{-T G(R, \tau)}
$$

where

$$
G(R, \tau)=\frac{1}{4 \pi \alpha^{\prime} \tau}+\left(\frac{R^{2}}{4 \pi \alpha^{\prime}}-\frac{(d-1) \pi}{24}\right) \tau+\frac{2 \pi}{4 \sqrt{2 \pi \alpha^{\prime} b}} .
$$

For $R$ above the critical distance

$$
R_{c}=\sqrt{\frac{\pi(d-1) 2 \pi \alpha^{\prime}}{12}}
$$

the equation

$$
-\frac{1}{4 \pi \alpha^{\prime} \tau^{2}}+\frac{R^{2}}{4 \pi \alpha^{\prime}}-\frac{(d-1) \pi}{24}=0
$$

has a solution

$$
\tau_{\min }(R)=\left(R^{2}-R_{c}^{2}\right)^{-1 / 2}
$$

which is an absolute minimum of the function $G(R, \tau)$. It follows that the large $T$ asymptotic of the integral (38) is given by the saddle point approximation

$$
W\left[c_{R T}\right]_{T \rightarrow \infty}^{--\longrightarrow} \mathrm{e}^{-T V(R)}
$$

where

$$
V(R)=\frac{1}{2 \pi \alpha^{\prime}} \sqrt{R^{2}-R_{c}^{2}}+\frac{\pi}{2 \sqrt{2 \pi \alpha^{\prime} b}}
$$

According to 22 $V(R)$ can be interpreted as the static quark potential in the Polyakov string approximation of QCD. The special feature of the Polyakov string is the appearance of a nonvanishing perimeter term.

\section{Semiclassical Approximation for $\mu>0$}

The approximation scheme developed in the previous section can be also applied to the functional integral (22). As before one can expand around $\phi_{n m} \equiv 0$. In the present case this is not a minimum of the whole action, but since the Liouville term is bounded from below the main contribution to the integral (22) comes from 
a small neighbourhood of the minimum of the "potential" term. Thus, expanding the logarithm and the exponential function one gets

$$
Z_{1 \text {-loop }}(R, T ; t) \approx e^{-\frac{1}{2 \epsilon^{2}}\left(\frac{T^{2}}{R^{2} t}+t\right)} \int \prod_{n, m \geq 0}^{\prime} d \phi_{n m} e^{-S_{1-\mathrm{loop}}[\phi]},
$$

where

$$
\begin{aligned}
S_{\text {1-loop }}[\phi] & =\frac{\epsilon^{2} b}{2}\left(2 \sum_{m \text { or } n=0}+\sum_{n, m \geq 1}\right)\left[\left(\frac{n^{2}}{t^{2}}+m^{2}+\frac{c}{b t}\right) \phi_{n m}{ }^{2}\right] \\
& +\frac{2 \epsilon c}{\sqrt{t}} \sum_{n, m \text { even }}{ }^{\prime} \phi_{n m} \\
& +\frac{T^{2}}{2 t R^{2}} \sum_{n=1}^{\infty}\left[\frac{\tanh \frac{\pi n}{2 t}}{\pi n}\left(\sum_{m \text { even }} \phi_{n m}\right)^{2}+\frac{\operatorname{coth} \frac{\pi n}{2 t}}{\pi n}\left(\sum_{m \text { odd }} \phi_{n m}\right)^{2}\right] \\
& +\frac{1}{2 t} \sum_{m=1}^{\infty}\left[\frac{\tanh \frac{\pi m t}{2}}{\pi m}\left(\sum_{n \text { even }} \phi_{n m}\right)^{2}+\frac{\operatorname{coth} \frac{\pi m t}{2}}{\pi m}\left(\sum_{n \text { odd }} \phi_{n m}\right)^{2}\right] .
\end{aligned}
$$

As before the conformal anomaly (the second factor in the equation above does not contribute to the result in the limit $\frac{T}{R} \rightarrow \infty$ and the last sum can also be neglected. Hence, for large $\frac{T}{R}$ the 1-loop effective action takes the form

$$
\begin{aligned}
S_{1 \text {-loop }}^{\prime}[\phi] & =\frac{\epsilon^{2} b}{2}\left(2 \sum_{m \text { or } n=0}+\sum_{n, m \geq 1}\right)\left[\left(\frac{n^{2} \pi^{2}}{t^{2}}+m^{2}+\frac{c}{b t}\right) \phi_{n m}{ }^{2}\right] \\
& +\frac{T^{2}}{2 t R^{2}} \sum_{n=1}^{\infty}\left[\frac{\tanh \frac{\pi n}{2 t}}{\pi n}\left(\sum_{m \text { even }} \phi_{n m}\right)^{2}+\frac{\operatorname{coth} \frac{\pi n}{2 t}}{\pi n}\left(\sum_{m \text { odd }} \phi_{n m}\right)^{2}\right] .
\end{aligned}
$$

Using the result (30) and the formula

$$
\operatorname{det}\left(\Delta+M^{2}\right)=\operatorname{det}(\Delta) \mathrm{e}^{\ln \delta \times M^{2} \int d^{2} z \sqrt{g}}
$$

we get (up to a constant)

$$
Z_{1 \text {-loop }}(R, T ; t) \approx e^{-\frac{1}{2 \epsilon^{2}}\left(\frac{T^{2}}{R^{2} t}+t\right)} \times I \times I_{o} \times I_{e},
$$

where

$$
\begin{aligned}
I & =t^{-\frac{1}{2}} \eta(t)^{-\frac{1}{2}} \\
\ln I_{o} & =-\frac{1}{2} \sum_{n} \ln \left(1+\frac{T^{2} \operatorname{coth} \frac{\pi n}{2 t}}{4 \epsilon^{2} b R^{2} n t} \frac{\tanh \frac{\pi}{2} \sqrt{\frac{n^{2}}{t^{2}}+\frac{c}{b t}}}{\sqrt{\frac{n^{2}}{t^{2}}+\frac{c}{b t}}}\right), \\
\ln I_{e} & =-\frac{1}{2} \sum_{n} \ln \left(1+\frac{T^{2} \tanh \frac{\pi n}{2 t}}{4 \epsilon^{2} b R^{2} n t} \frac{\operatorname{coth} \frac{\pi}{2} \sqrt{\frac{n^{2}}{t^{2}}+\frac{c}{b t}}}{\sqrt{\frac{n^{2}}{t^{2}}+\frac{c}{b t}}}\right)
\end{aligned}
$$

The series above are convergent for all positive values of parameters $\frac{T}{\epsilon R}, t$. Summing over $n$ from 1 to $\sqrt{t}$ and from $\sqrt{t}$ to $\infty$ and using the formula (35) one gets for $\frac{t}{T}=$ const, $T \rightarrow \infty$

$$
\ln I_{o}=\ln I_{e}=-\frac{\pi T}{4 \sqrt{b} \epsilon R}+O\left(\sqrt{T} \ln \frac{\pi T}{\sqrt{b} \epsilon R}\right) .
$$


Inserting (45) into the formula (44) one obtains

$Z_{1 \text { loop }}(R, T ; t) \approx t^{-\frac{1}{2}} \eta(t)^{-\frac{1}{2}} \exp \left[-\frac{1}{\epsilon^{2}}\left(\frac{T^{2}}{2 R^{2} t}+\frac{t}{2}+\epsilon \frac{2 \pi T}{4 \sqrt{b} R}+\epsilon^{2} O\left(\sqrt{T} \ln \frac{T}{\epsilon R}\right)\right)\right]$.

The formula above exactly coincides with the corresponding one (37) derived in the case $\mu=0$. Thus repeating all the calculations of the previous section one arrives at the result (41).

\section{Conclusions}

The comparison of our results with the predictions of the string models from Lüscher's universality class [6] shows two differences. The first one consists in the presence of the nonvanishing "universal" perimeter term. The second more important feature is that the longitudinal mode of noncritical Polyakov string manifests itself simply as an additional degree of freedom. This can be easily seen comparing the formulae (39,41) with the result found in the Nambu-Goto string model by Alvarez [8] and Arvis [9].

$$
\begin{aligned}
R_{c}^{\mathrm{N}-\mathrm{G}} & =\sqrt{\frac{\pi(d-2) 2 \pi \alpha^{\prime}}{12}}, \\
V^{\mathrm{N}-\mathrm{G}}(R) & =\frac{1}{2 \pi \alpha^{\prime}} \sqrt{R^{2}-R_{c}^{\mathrm{N}-\mathrm{G}^{2}}} .
\end{aligned}
$$

Our semiclassical result leads to the conclusion that the Polyakov sum over bordered surfaces does not provide a good ansatz for the Wilson loop. This is especially striking in two dimensions where the formula (41) essentially differs from the linear static potential predicted by QCD.

In string theory the static potential $V(R)$ is interpreted as the ground state energy of the string with ends fixed at the distance $R$. In the semiclassical approximation the expressions for the rectangular Wilson loops (now interpreted as a string propagator) are different for $\mu=0$ and $\mu>0$. Nevertheless, the large $T$ asymptotics exactly coincide. A closer analysis shows that not only the ground state energies but the whole spectra are identical. This result is consistent with the conjecture [27] that the Liouville theory may be a complicated off-shell extension of a basically simple model.

The investigations of the static potential presented in this paper leave the open problem of higher loop corrections. A more detailed analysis of this point is in fact necessary for a full justification of the assumption made in our calculations. It is also interesting to understand the structure of the nonstandard loop expansion introduced in Section 4. The 1-loop calculations suggest that it is quite regular. In fact the only renormalization we need in the gaussian models (29), (43) generating the loop expansions concerns the "bulk" part of the determinant in the formulae (31) and (44) respectively..

The question arises how general the methods employed in this paper are. The perturbation method introduced in the beginning of Sect.4 is based on the assumption that for each value of the Teichmüller parameter $t \in R_{+}$, there exists a minimum of the functional $S_{\text {eff }}\left[c, e^{\phi} \widehat{g}_{t}\right]$ (11). In the general case of a model surface $M$ with a boundary $\partial M$ mapped to a given contour $c \subset R^{d}$ by $\widetilde{c}: \partial M \rightarrow c$ the corresponding functional $S[\widetilde{c}, \widetilde{\phi} ; t]$ can be regarded as a functional $S[\widetilde{c}, \gamma ; t]$ on the space $\operatorname{Diff}(\partial M) \times T_{M}$ where $T_{M}$ is the Teichmüller space of the surface $M$. Note that 
$S[\widetilde{c}, \gamma ; t]$ is just the energy functional from the theory of harmonic maps [28] evaluated at the solution $x_{c l}$ of the Dirichlet boundary problem $\Delta_{t} x_{c l}=0, x_{c l \mid \partial M}=\widetilde{c} \circ \gamma$. The minimal parametrization $\gamma_{\min }(t)$ is then uniquely determined by the solution of the free boundary problem for the contour $c$. Sufficient conditions for the existence of solution to this problem are usually expressed in terms of some regularity conditions for the contour $c$ [28]. Now, the existence of a minimum of the function $S\left[\widetilde{c}, \gamma_{\min }(t) ; t\right]$ on $T_{M}$ is equivalent to the existence of a solution of the Plateau problem (with the fixed topological type) [28]. The resulting conditions for the contour $c$ precisely coincide with that one encounters in the semiclassical calculations in the Schild-Eguchi string model [5]. The existence of a minimum of the interaction term provides merely a justification for the perturbation scheme developed in Sect.4. For concrete calculations one needs an explicit solution of the free boundary problem for each point in the Teichmüller space. This is the main technical limitation of the semiclassical calculations in string models. Nevertheless, using the methods developed in the case of long rectangular loop one can address all the questions of the program initiated in Ref. [5].

As was mentioned in the introduction, semiclassical calculations of the static potential can be used as the simplest test for any definition of the sum over surfaces. In the case of the conceptually simple but complicated theory formulated in Sect.2 this test yields surprisingly good results. This leads us to the main conclusion of this paper that in the range $1<d<25$ the model derived in Sect.2 is a good candidate for a well defined sum over surfaces in the continuum formulation. It seems that the program of semiclassical calculations sketched above can provide some additional information about the quantum theory of conformal factor and in particular the relevance of the Liouville interaction. However, the most intriguing question is whether the sum over surfaces yields a consistent quantum theory of noncritical string. The work on this problem is in progress and will be presented elsewhere.

\section{Acknowledgements}

One of us (Z.J.) is grateful to Professor A.Verjovsky for enlightening discussions on the variational problems related to this work. We would like to thank Professor Abdus Salam, the International Atomic Energy Agency and UNESCO for support during our stay at the International Centre of Theoretical Physics where this work was carried out.

\section{References}

[1] A.M.Polyakov, Phys.Lett. 103B (1981) 207.

[2] K.Wilson, Phys.Rev. D10 (1974) 2445.

[3] Y.Nambu, Phys.Lett. 80B (1979) 375

J.L.Gervais, A.Neveu, Phys.Lett. 80B (1979) 255

A.M.Polyakov, Phys.Lett. 82B (1979) 247

M.Lüscher, Phys.Lett. 90B (1980) 277.

[4] A.Schild, Phys.Rev. D16 (1977) 1722

T.Eguchi, Phys.Rev.Lett. 44 (1980) 126. 
[5] M.Lüscher, K.Symanzik, P.Weisz, Nucl.Phys. B173 (1980) 365.

[6] M.Lüscher, Nucl.Phys. B180 [FS2] (1981) 317.

[7] K.Dietz, T.Filk, Phys.Rev. D27 (1983) 2944.

[8] O.Alvarez, Phys.Rev. D24 (1981) 440.

[9] J.F.Arvis, Phys.Lett. 127B (1983) 106.

[10] J.Ambjorn, P.Olesen, C.Peterson, Nucl.Phys. B244 (1984) 262

C.Peterson, L.Sköld, Nucl.Phys. B255 (1985) 365

D.Barkai, K.J.M.Moriarty, C.Rebbi, Phys.Rev. D30 (1984) 1293

S.W.Otto, J.D.Stack, Phys.Rev.Lett. 52 (1984) 2328

Ph. de Forcrand, G.Schierholz, H.Schneider, M.Teper, Phys.Lett. 160B (1985) 137

H.-Q.Ding, C.F.Baillie, G.C.Fox Phys.Rev. D41 (1990) 2912

H.-Q.Ding, Phys.Rev. D24 (1990) 2350

G.S.Bali, K.Schilling Bergische Universität preprint (1992) WUB 92-02.

[11] M.Flensburg, C.Peterson, Phys.Lett. 153B (1985) 412 ; Nucl.Phys. B283 (1987) 141

M.Caselle, R.Fiore, F.Glozzi, Phys.Lett. 224B (1989) 153

M.Gao, Phys.Lett. 244B (1990) 488

H.-Q.Ding, Phys.Rev. D44 (1991) 2200.

[12] P.Olesen, Phys.Lett. 160B (1985) 144.

[13] S.N.Gupta, S.F.Radford, W.W.Repko, Phys.Rev. D26 (1982) 3305; 34 (1986) 201.

[14] O.Alvarez, Nucl.Phys. B216 (1983) 125.

[15] E.S.Fradkin, A.A.Tseytlin, Ann.Phys.(N.Y.) 143 (1982) 413.

[16] B.Durhuus, P.Olesen, J.L.Petersen, Nucl.Phys. B232 (1984) 291.

[17] F.David, Mod.Phys.Lett. A3 (1988) 1651.

[18] J.Distler, K.Kawai, Nucl.Phys. B321 (1989) 509.

[19] N.Mavromatos, J.Miramontes, Mod.Phys.Lett. A4 (1989) 1849.

[20] E.D'Hoker, P.S.Kurzepa, Mod.Phys.Lett. A5 (1990) 1411.

[21] A.Cohen, G.Moore, P.Nelson, J.Polchinski, Nucl.Phys. B267 (1986) 143.

[22] Z.Jaskólski, Commun.Math.Phys. 128 (1990) 285.

[23] Z.Jaskólski, Int.J.Mod.Phys. A6 (1993) 1041.

[24] Z.Jaskólski, Commun.Math.Phys. 139 (1991) 353.

[25] C.Varughese, W.I.Weisberger, Phys.Rev. D37 (1988) 981.

[26] N.Seiberg, Notes on quantum Liouville theory and quantum gravity, Rutgers preprint RU-90-29 (1990).

[27] E.D'Hoker, Lecture notes on 2-d gravity and Liouville theory, University of California preprint UCLA/91/TEP/35 (1991).

[28] J.Jost, Two-dimensional geometric variational problems, Wiley - Interscience, Chichester, (1991).

[29] J.Jost, Strings with boundary : a quantization of Plateau's problem, SFB 237 preprint (1988). 


\section{Appendix A}

In this Appendix we will prove that the corner anomaly does not give any contribution to the quark potential in the limit $R / T \rightarrow 0$.

Let us consider the even-even sector (the only one where the corner enters).

$$
\begin{aligned}
S_{\mathrm{ee}} & =\frac{b}{2}\left(2 \sum_{m \text { or } n=0}+\sum_{n, m \geq 2}\right)_{m, n \text { even }}\left[\left(\frac{n^{2}}{t^{2}}+m^{2}\right) \phi_{n m^{2}}{ }^{2}\right] \\
& +\frac{T^{2}}{4 \pi \alpha^{\prime} \pi t} \sum_{n \text { even } \geq 2} \frac{\tanh \frac{\pi n}{2 t}}{n}\left(\sum_{m \text { even }} \phi_{n m}\right)^{2}+\frac{c}{\sqrt{t}} \sum_{m n \text { even }} \phi_{n m} .
\end{aligned}
$$

We use the well known formula for the Gaussian functional integration:

$$
\int \prod_{i} d \phi_{i} \exp \left(-\phi_{i} A_{i j} \phi_{j}+b_{i} \phi_{i}\right)=\text { const }(\operatorname{det} A)^{-1 / 2} \exp \left(b_{i} A_{i j}^{-1} b_{j} / 4\right) .
$$

The determinant has been considered in the main text so we will take into account only the argument of the exponential. As before we can treat separately each $\mathrm{n}$ and sum over it at the end. We have to remember that $m$ and $n$ run over even integers only.

It is easy to prove

$$
\begin{gathered}
\frac{1}{4} b_{i} A_{i j}^{-1} b_{j}=\frac{c^{2}}{4 t}\left(\begin{array}{lllll}
1 & 1 & \ldots & 1
\end{array}\right)\left(\begin{array}{cccc}
\alpha_{1}+\beta & \beta & \ldots & \beta \\
\beta & \alpha_{2}+\beta & \ldots & \beta \\
\ldots & \ldots & \ldots & \ldots \\
\beta & \beta & \ldots & \alpha_{n}+\beta
\end{array}\right)^{-1}\left(\begin{array}{c}
1 \\
1 \\
\ldots \\
1
\end{array}\right) \\
=\frac{c^{2} \frac{\sum \alpha_{m}^{-1}}{4 t} \frac{}{1+\beta \sum \alpha_{m}^{-1}}}{}
\end{gathered}
$$

where

$$
\begin{gathered}
\alpha_{m}=\frac{b}{2}\left(\frac{n^{2}}{t^{2}}+m^{2}\right), \alpha_{0}=\frac{b n^{2}}{t^{2}} \\
\beta=\frac{T^{2} \tanh \frac{\pi n}{2 t}}{4 \pi \alpha^{\prime} \pi t n} .
\end{gathered}
$$

Using the equation (32) we get the total result (we changed $n$ into $n / 2$ so that now $n$ runs over all positive integers)

$$
-\frac{c^{2}}{16 b t} \sum_{n=1}^{\infty} \frac{\frac{\pi n}{t} \operatorname{coth} \frac{\pi n}{t} \mathrm{e}^{-\delta n^{2} / t^{2}}}{\frac{n^{2}}{t^{2}}+\frac{T^{2}}{32 \pi \alpha^{\prime} b t^{2}}}
$$

We have introduced the regularization by the kinetic term - after removing the regulator the result in the limit $T \rightarrow \infty, t / T=$ const is just a function of $t / T$ and not $T \times$ function $(t / T)$ so the whole contribution from the anomaly on the corners to the potential can be neglected.

At the end we would like to justify the fact that in the limit $R / T \rightarrow 0$ we dropped the infinite sum proportional to $R^{2}$ in eq. (28). The structure of the eq. (28) can be presented in the following form:

$$
A / T^{2}=A_{0}+\frac{R^{2}}{T^{2}} \delta A=\left(\begin{array}{cccc}
A_{11 m m^{\prime}} & 0 & 0 & \ldots \\
0 & A_{22 m m^{\prime}} & 0 & \ldots \\
\ldots & \ldots & \ldots & \ldots
\end{array}\right)
$$




$$
+\frac{R^{2}}{T^{2}}\left(\begin{array}{cccccc}
0 & 0 & A_{13 m m^{\prime}} & 0 & A_{15 m m^{\prime}} & \ldots \\
0 & 0 & 0 & A_{24 m m^{\prime}} & 0 & \ldots \\
A_{31 m m^{\prime}} & 0 & 0 & 0 & A_{35 m m^{\prime}} & \ldots \\
\ldots & \ldots & \ldots & \ldots & \ldots & \ldots
\end{array}\right)
$$

where each entry with given values of $n n^{\prime}$ corresponds to an infinite matrix in $\mathrm{mm}^{\prime}$. We expand in $R^{2}$ the determinant of $A$ :

$$
\ln \operatorname{det} A \approx \ln \operatorname{det} A_{0}+\frac{R^{2}}{T^{2}} \operatorname{Tr}\left(A_{0}^{-1} \delta A\right)
$$

The first term was considered in the main text while the second (which is anyway proportional to $R^{2} / T^{2}$ ) vanishes since $\delta A$ is off-diagonal. It means that we can safely drop the whole $R^{2}$ correction to the determinant of $A$. 